\title{
Periorbitale Ulzeration
}

\section{Herpes? Lues? Neoplasie?}

Teresa Jaeger, Sabine G. Plötz, Soja Shamizadeh, Michael Zirbs, Johannes Ring, Rüdiger Hein, Markus Ollert, Christian ANDRES

\begin{abstract}
Eine 31-jährige, ansonsten gesunde Patientin stellte sich bei uns mit einer seit ca. vier Wochen bestehenden Ulzeration im Bereich des linken Auges vor. Differenzialdiagnostisch wurde an ein Ulcus durum (Lues I), eine Herpesvirus-bedingte Ulzeration, eine Tinea profunda, ein Keratoakanthom sowie an ein Artefakt gedacht.
\end{abstract}

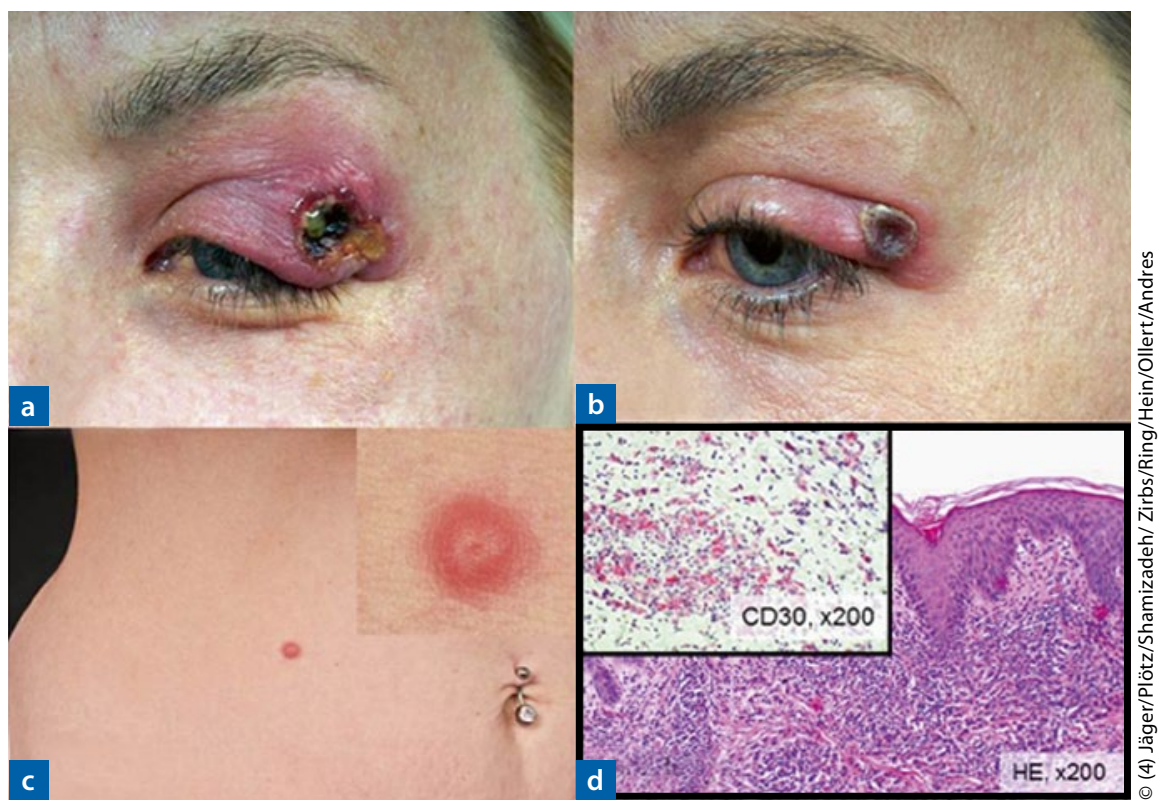

[ ine pragmatische Therapie mit sys-

- temischen Antibiotika (Cefuroxim, Clarithromycin) und Virostatika (Aciclovir) zeigte nur wenig Ansprechen (Abb. 1b) und es kam im Verlauf zum Auftreten von erythematösen, papulonodulären Hautläsionen am Integument (Abb. 1c).

Es erfolgte nun eine 4-mm-Hautbiopsie. Die Histologie zeigte ein dichtes gemischtzelliges Infiltrat in der der oberen Dermis u. a. mit Beimengung von Eosinophilen. Die Lymphozyten waren zum Teil CD30-positiv (Abb. 1d).

\section{Diagnose}

In Zusammenschau mit dem klinischen Verlauf wurde die Diagnose einer lym- phomatoiden Papulose (Typ A) gestellt. Es erfolgte eine lokale Therapie mit Clobethasol-Creme, des Weiteren eine Creme-PUVA (Psoralen-UVA-Lichttherapie), woraufhin sich ein gutes Ansprechen zeigte.

\section{Diskussion}

Die lymphomatoide Papulose gehört zur Gruppe der primär kutanen CD30+ lymphoproliferativen Erkrankungen, wobei vier histologische Subtypen unterschieden werden. Am häufigsten findet sich Typ A mit einem gemischtzelligen Infiltrat aus CD30-positiven Lymphozyten, Eosinophilen und Neutrophilen. In Typ B zeigt sich eine Population von kleinen Lymphozyten (CD30-positiv oder CD30-
Abb. 1: Ulzeration des linken Auges (a) mit wenig Ansprechen auf systemische Antibiotika und Virostatika (b); im weiteren Verlauf Hinzutreten von papulonodulären Hautveränderungen am Integument (c); gemischtzelliges Infiltrat u.a. mit Beimengung von Eosinophilen, zum Teil CD30-positive Lymphozyten (d).

negativ) mit Epidermotropismus, ähnlich der Histologie einer Mycosis fungoides. Typ C besteht aus großen CD30-positiven anaplastischen Zellen und darf nicht mit dem anaplastischen großzelligen T-Zell-Lymphom verwechselt werden. Typ D ist histologisch nur schwer vom primären kutanen aggressiven epidermotropischen CD8+ zytotoxischen TZell Lymphom zu unterscheiden.

Die Prognose der lymphomatoiden Papulose ist sehr gut. Der Verlauf kann über Jahre gehen, jedoch kann es auch innerhalb von Wochen oder Monaten zur spontanen Regression kommen. Dennoch sollte ein Monitoring der Patienten erfolgen, da es in seltenen Fällen zum Übergang in eine Mycosis fungoides, ein Hodgkin-Lymphom oder ein anaplastisches T-Zell-Lymphom kommen kann (4-25\%).

Die Patienten profitieren aufgrund des Verlaufes nicht von einer aggressiven Therapie. Mittel der Wahl sind topische Steroide, Lichttherapie (Psoralen-UVA) sowie ggf. Methotrexat.

\section{Dr. med. Teresa Jaeger}

Klinik und Poliklinik für Dermatologie und

Allergologie der TU München

Biedersteiner Str. 29

80802 München 